\title{
Unsaturated fatty acids
}

\author{
Helen M. Roche \\ Unit of Nutrition and Dietetics, Trinity Centre for Health Sciences, St James's Hospital, James's Street, Dublin 8 , \\ Republic of Ireland
}

\begin{abstract}
There is good scientific evidence that dietary fatty acid composition is involved in the aetiology of many diseases. Increasing the supply of $n-3$ polyunsaturated fatty acids (PUFA) may reduce the risk of CHD. Several scientific organizations (for example, see Department of Health, 1991, 1994; British Nutrition Foundation, 1992; Scientific Committee for Food, 1993; Food and Agriculture Organization/World Health Organization, 1998) have made recommendations for $n-3$ PUFA; however, there is a high degree of variation both in terms of the type and amount of $n$ - 3 PUFA (up to 7-fold). This variation reflects the different scientific axioms which underlie the different recommendations. Optimal nutrition may be defined in terms of the level of a nutrient required to avoid deficiency, or the amount required to have an effect on biomarkers and functional indicators of nutrient intake, or the level of a nutrient which prevents disease. Functional biomarkers of $n-3$ PUFA include plasma, platelet and erythrocyte phospholipid- $n-3$ PUFA levels. Plasma triacylglycerol concentrations represent a functional indicator of $n-3$ PUFA because $n-3$ PUFA exert a consistent hypotriacylglycerolaemic effect which is dose-dependent and persistent. In terms of disease status, epidemiological studies have demonstrated that the incidence of CHD is inversely associated with consumption of $n$ - 3 PUFA. Despite the health benefits of $n$ - 3 PUFA, the mean daily intake falls far short of most of the recommendations. Increasing fish intake is the most obvious way to increase $n$-3 PUFA intake. However, a large percentage (up to 65) of the population do not eat fish. Thus, there is a need for alternative sources of $n-3$ PUFA, such as functional foods, whose unique fatty acid composition could fortify staple foods thereby promoting optimal levels of $n$-3 PUFA intake.
\end{abstract}

Dietary fat: $n$-3 PUFA: Triacylglycerol: Coronary heart disease

Dietary fat is an essential nutrient providing energy, essential fatty acids and fat-soluble vitamins. Whilst adequate amounts of dietary fat are readily provided by the Westerntype diet, the optimal fatty acid composition of the diet is an important factor which can play a role in disease prevention and promote health. The Joint Food and Agriculture Organization/WHO Expert Consultation on Fats and Oils in Human Nutrition (Food and Agriculture Organization/ World Health Organization (FAO/WHO), 1998) concluded that 'Adequate amounts of dietary fat are essential for health. In addition to their contribution to meeting energy needs, intakes of dietary fat must be sufficient to meet requirements for essential fatty acids and fat-soluble vitamins'. In developing countries it is important that adequate amounts of dietary fat are supplied to prevent deficiency. However, in the Western hemisphere more than adequate, if not excessive, quantities of fat are supplied by the diet, and nutritional concern relates more to the optimal dietary fatty acid composition. For example, there is a growing body of scientific evidence that increasing the supply of unsaturated fatty acids, especially $n-3$ polyunsaturated fatty acids (PUFA) may be required to reduce the risk of disease, especially CHD.

In order to define the optimal level of dietary unsaturated fatty acid intake it is important to realize that fatty acids have a wide range of biological roles and cellular functions. Fatty acids are an integral component of the phospholipid bilayer of cellular membranes, which in turn affects membrane fluidity and lipid-protein interactions. Thus fatty acids can affect transport proteins and cellular receptors for hormones and neurotransmitters. Fatty acids are the substrates for eicosanoid synthesis, and fatty acids also affect the production of many biologically-active compounds, including lipid-derived cellular mediators such as platelet-activating factor, and cytokines. Fatty acids also have the ability to affect the expression of genes encoding

\footnotetext{
Abbreviations: ALA, $\alpha$-linolenic acid; BNF, British Nutrition Foundation; COMA, Committee on Medical Aspects of Food Policy; DHA, docosahexaenoic acid; EPA, eicosapentaenoic acid; FAO/WHO, Food and Agriculture Organization/WHO; PUFA, polyunsaturated fatty acids; SCF, Scientific Committee for Food; TAG, triacylglycerols.

Corresponding author: Dr Helen Roche, fax +3531 4542043, email hmroche@tcd.ie
} 
for enzymes which are involved in lipid metabolism and to interact with nuclear receptor proteins that bind to DNA, therefore fatty acids can alter the transcription of regulatory genes. With this wide range of functions in mind, the present paper will attempt to define optimal levels of dietary unsaturated fatty acid intake in terms of biomarkers and functional indicators of dietary intake, in order to determine the optimal levels of these nutrients which are required for disease prevention.

\section{Structure and nomenclature of fatty acids}

Fatty acids are composed of a hydrocarbon chain with a methyl group and a terminal carboxyl group. The properties of dietary fat are primarily determined by the composition of its fatty acids, which may be saturated (no double bonds), monounsaturated (one double bond) or PUFA (greater than one double bond). Unsaturated fatty acids can adopt two distinct geometric configurations, denoted as the cis and trans configurations. Cis double bonds have the two hydrogen atoms on the same side of the molecule, whilst in the trans configuration they are on opposite sides of the molecule. Geometrical isomerism has implications for the shape and physical properties of the molecule, whereby cisfatty acids have a kink in the chain and trans-fatty acids adopt a straight configuration and function like saturated fatty acids.

PUFA may be subdivided into two categories, the $n-6$ and $n-3$ series, depending on whether the double bond closest to the non-carboxyl end is located at the C- 6 or C-3 respectively. Human subjects are unable to synthesize $n-6$ and $n-3$ PUFA, therefore these fatty acids are essential fatty acids which must be supplied by the diet. Linoleic acid (18:2n-6) and $\alpha$-linolenic acid (ALA; $18: 3 n-3$ ) are the principal essential unsaturated fatty acids. These fatty acids can be elongated and desaturated into their longer-chain derivatives arachidonic acid (20:4n-6), eicosapentaenoic acid (EPA; 20:5n-3) and docosahexaenoic acid (DHA; 22:6n-3).

\section{Recommendations for optimal unsaturated fatty acid intake}

Recommendations for average population intakes for total and unsaturated fatty acids have been proposed by a number of scientific authorities, including the Committee on Medical Aspects of Food Policy (COMA; Department of
Health, 1991, 1994), the British Nutrition Foundation (BNF; 1992), the Scientific Committee for Food (SCF; 1993) and the FAO/WHO (1998). Table 1 demonstrates that similar intakes of total fat, monounsaturated fatty acid, $n$-6 PUFA and trans-fatty acids are being recommended by the different scientific authorities. Furthermore, the mean daily intake of these fatty acids by adults in the UK (Gregory et al. 1990) compares well with these recommendations.

However, it is important to note that there is a high degree of variation in the amount of $n-3$ PUFA which is recommended by the different organizations. Furthermore, the mean daily intake of $n$-3 PUFA (Gregory et al. 1990) falls far short of most of the recommendations. The specific recommendations for $n$-3 PUFA intake are detailed in Table 2. The recommendations vary in terms of both the type and amount of $n-3$ PUFA, and this reflects the different scientific axioms which underlie the different recommendations. For example, the SCF (1993) and FAO/WHO (1998) make recommendations for ALA only, on the understanding that human subjects have the enzymes to elongate and desaturate ALA to form EPA and DHA. Thus, according to the SCF (1993) and FAO/WHO (1998), EPA and DHA are not essential fatty acids and there are no recommendations for optimal levels of intake for these fatty acids. However, COMA (Department of Health, 1991) and the BNF (1992) recognize that this conversion process is relatively inefficient, and that there are added biological functions and health benefits which are exclusive to EPA and DHA (Freese \& Mutanen, 1997). Thus, the BNF (1992) considers EPA and DHA to be 'conditionally essential', and specific recommendations are made for EPA and DHA.

The scientific committees also use different units or measures to express their $n$-3 PUFA recommendations. For example, the BNF (1992) and the SCF (1993) express their recommendations as \% dietary energy, whilst COMA (Department of Health, 1991) uses two measures, \% dietary energy from ALA and an absolute amount for EPA (g/week). FAO/WHO (1998) base their recommendations for $n$-3 PUFA intake on the linoleic acid: ALA value. When all the recommendations are expressed in a similar fashion (as \% dietary energy) it is apparent that there is a great variation in the level of $n$-3 PUFA being recommended, ranging from $0.2 \%$ energy to $1.5 \%$ energy. This sevenfold difference not only reflects the different methods used to determine the recommendations, but it also reflects a different appreciation of the concept of optimal nutrition. COMA

Table 1. Recommendations for average population intakes of unsaturated fatty acids compared with current intakes in the UK (\% total energy)

\begin{tabular}{|c|c|c|c|c|c|}
\hline & Total fat & MUFA & $n-6$ PUFA & $n$-3 PUFA & trans-FA \\
\hline Department of Health (1994) & 33 & 12 & $>1 \cdot 0$ & $>0.2$ & $<2.0$ \\
\hline Scientific Committee for Food (1993) & - & - & $2 \cdot 0$ & 0.5 & - \\
\hline \multicolumn{6}{|l|}{ Food and Agriculture Organization/World } \\
\hline Health Organization (1998) & $15-30$ & $10-15$ & $4-10$ & $0.4-2 \cdot 0$ & - \\
\hline \multicolumn{6}{|l|}{ Current intakes (Gregory et al. 1990) } \\
\hline
\end{tabular}

MUFA, monosaturated fatty acids; PUFA, polyunsaturated fatty acids; FA, fatty acids. 
Table 2. Recommendations for $n-3$ polyunsaturated fatty acid (PUFA) intake

\begin{tabular}{lll}
\hline Reference & Specific recommendations for $n$-3 PUFA & $n$-3 PUFA (\% energy) \\
\hline Department of Health (1991, 1994) & At least of $0.2 \%$ energy from ALA & 0.2 \\
& $1.5 \mathrm{~g}$ EPA and DHA/week \\
British Nutrition Foundation (1992) & $1.0 \%$ Energy from ALA \\
& $0.5 \%$ Energy from EPA and DHA \\
Scientific Committee for Food (1993) & $0.5 \%$ Energy from $n-3$ PUFA \\
Food and Agriculture Organization/ & $4-10 \%$ Energy from LA \\
World Health Organization (1998) & LA: ALA 5:1-10:1 \\
& (using 7\% energy from LA and LA : ALA of 7.5:1) & 0.5 \\
\hline
\end{tabular}

ALA, $\alpha$-linolenic acid; DHA, docosahexaenoic acid; EPA, eicosapentaenoic acid; LA, linoleic acid.

(Department of Health, 1991) and the SCF (1993) make recommendations to prevent essential fatty acid deficiency. In contrast the BNF (1992) and the FAO/WHO (1998) recognize that there are additional beneficial effects associated with greater levels of intake of these fatty acids, therefore these recommendations are quantitatively greater to prevent deficiency and promote health.

\section{Defining optimal levels of $\boldsymbol{n}$-3 polyunsaturated fatty acid intake}

Optimal nutrition may be defined in terms of the level of a nutrient required to avoid deficiency, or the amount required to have an effect on biomarkers and functional indicators of nutrient intake, or the level of a nutrient which prevents disease. In terms of trying to define the optimal levels of $n-3$ PUFA intake a combination of these principles may be used. In terms of disease status, epidemiological studies have demonstrated that the incidence of CHD is inversely associated with consumption of $n$-3 PUFA (Bang \& Dyerberg, 1972; Hirai et al. 1980). The Zutphen Study (Kromhout et al. 1985) provided prospective evidence that there was an inverse relationship between fish consumption and 20-year mortality from CHD. The diet and reinfarction trial (Burr et al. 1989) demonstrated that relatively low doses of $n-3$ PUFA (2.3 g EPA/week, which is equivalent to two to three portions of oily fish every week), reduced the risk of secondary coronary events. It has also been demonstrated that the Mediterranean ALA-rich diet is associated with a significant reduction in the number of non-fatal myocardial infarctions and cardiac deaths (de Lorgeril et al. 1994). A recent prospective study in patients with acute myocardial infarction has demonstrated that supplementation with a low dose of fish oil (1.08 g EPA/d) and mustard-seed oil $(2.9 \mathrm{~g}$ ALA/d) for 1 year was associated with a significant reduction in the incidence of cardiac events (Singh et al. 1997). In that study, the total number of cardiac deaths was significantly reduced by fish oil but not by the mustard-seed-oil supplement, thus illustrating that ALA and the long-chain $n$-3 PUFA may have different levels of nutritional efficacy.

Numerous intervention studies have demonstrated that $n$-3 PUFA exert their cardio-protective effects in a variety of ways, including anti-thrombotic (Leaf \& Weber, 1988), anti-arrhythmic (Kim et al. 1995) and anti-inflammatory (Endres et al. 1989; Wallace et al. 1995) effects, as well as improved plasma lipid and lipoprotein concentrations
(Roche \& Gibney, 1996). In terms of defining optimal levels of unsaturated fatty acid intake, $n-3$ PUFA lend themselves well to the concept of the use of biomarkers or functional indicators of $n-3$ PUFA intake. As a biomarker there is a strong positive relationship between the amount of $n-3$ PUFA consumed and the levels of EPA and DHA which become incorporated into plasma, platelet and erythrocyte phospholipids (Katan et al. 1991; Li \& Steiner, 1991; Roche \& Gibney, 1994). Thus, the levels of EPA and DHA in cellular membranes are an accurate measure of actual dietary fish and fish oil intake (Agren et al. 1990; Anderson et al. 1996). Dietary ALA intake has a strong positive relationship with plasma and neutrophil phospholipid-ALA and -EPA (Mantzioris et al. 1995).

There is a functional relationship between $n-3$ PUFA intake and plasma triacylglycerol (TAG) concentrations. In human subjects $n$-3 PUFA exert a consistent hypotriacylglycerolaemic effect, which is dose-dependent and persistent. In a recent review of seventy-two placebo-controlled human studies which provided supplemental EPA and DHA in the range $1 \cdot 0-7 \cdot 0 \mathrm{~g} / \mathrm{d}$ for at least 2 weeks, plasma TAG concentrations were consistently reduced by $25-30 \%$ (Harris, 1996). Several studies have shown that fish-oil supplementation has a dose-dependent hypotriacylglycerolaemic effect on fasting plasma TAG concentrations (Sanders et al. 1981; Blonk et al. 1990; Schmidt et al. 1990). When the data from these studies are pooled (Fig. 1), it emerges that the change ( $\%$ baseline concentration) in fasting plasma TAG concentration $(\Delta \mathrm{T})$ is related to the $n-3$ PUFA intake ( $\mathrm{g} n-3$ PUFA) according to the equation $\Delta \mathrm{T}=$ $-7 \cdot 67-3 \cdot 05$ (g $n-3$ PUFA) $\left(R^{2} 0 \cdot 87\right)$. It is important to note that this equation relates to doses of $n-3$ PUFA ranging between 1 and $9 \mathrm{~g} / \mathrm{d}$, which were added to the diet for a relatively short period of time (4-12 weeks). It is also important to note that the duration of supplementation also affects the hypotriacylglycerolaemic efficacy of $n-3$ PUFA. The reduction in plasma TAG concentrations demonstrated in long-term supplementation trials using low doses of $n-3$ PUFA was equivalent to that in short-term high-dose $n-3$ PUFA supplementation trials. Investigations by our research group (Roche \& Gibney, 1996) have demonstrated that a low dose of fish oil ( $1 \mathrm{~g} n$-3 PUFA/d) given as a supplement for 16 weeks significantly $(P<0.05)$ reduced fasting plasma TAG concentration by $21.2 \%$, a level much greater than that predicted from the previously described equation $(-10.7 \%)$. 


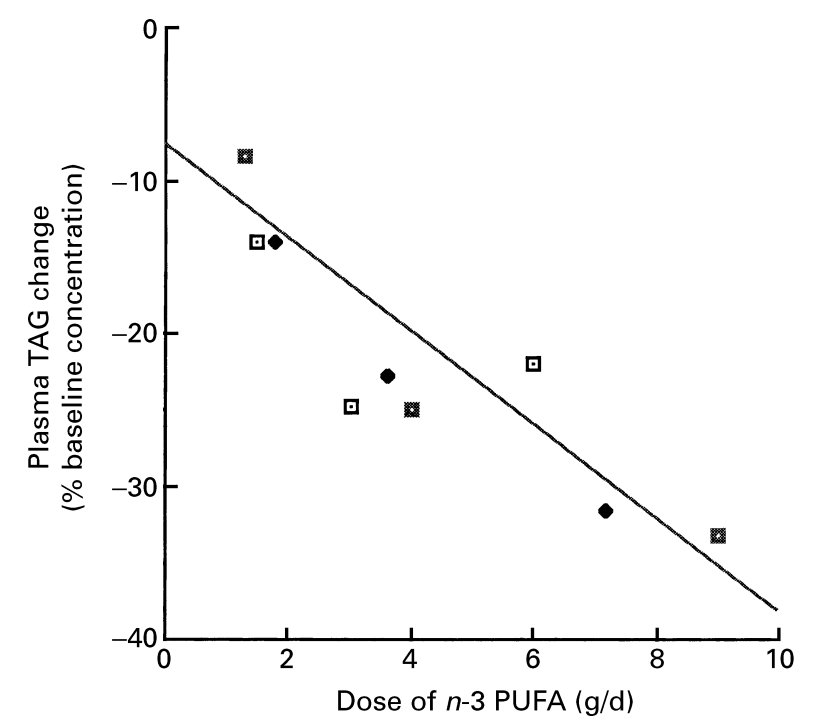

Fig. 1. Dose-dependent hypotriacylglycerolaemic effect of $n-3$ polyunsaturated fatty acids (PUFA). (๑), Blonk et al. (1990); ( ), Sanders et al. (1983); (a), Schmidt et al. (1990). TAG, triacylglycerols. Regression equation: $y=-7.67-3.05 x ; R^{2} 0.87$. (Adapted from Roche \& Gibney, 1996.)

\section{The relative efficacy of $\boldsymbol{n - 3}$ polyunsaturated fatty acids}

Whilst discussing the use of biomarkers and functional indicators of $n-3$ PUFA intake it is important to note that ALA and the long-chain $n$-3 PUFA have different effects. ALA supplementation significantly increases the level of ALA and EPA in plasma and cells, but ALA supplementation does not increase DHA or reduce arachidonic acid levels (Sanders \& Younger, 1981). This is an important difference because the balance of arachidonic acid and EPA determines the type and biological efficacy of eicosanoids, which in turn controls thrombosis and the immune and inflammatory responses. It has been demonstrated that both ALA and long-chain $n$-3 PUFA supplementation have equivalent effects on haemostatic factors (Freese \& Mutanen, 1997). However, unlike EPA and DHA, ALA supplementation has no effect on plasma TAG concentrations (Sanders et al. 1989; Freese \& Mutanen, 1997). There are also differences between ALA and the long-chain $n$-3 PUFA in terms of disease outcome. Singh et al. (1997) demonstrated that whilst ALA supplementation was as efficacious as fish oil in reducing the incidence of cardiac events, EPA and DHA but not ALA significantly reduced the number of total cardiac deaths.

\section{Current dietary intake $v$. optimal levels of $n-3$ polyunsaturated fatty acid consumption}

It is difficult to quantify the actual intake of $n-3$ PUFA across Europe; with the exception of the dietary survey in the UK (Gregory et al. 1990), no national survey has quantified $n$-3 PUFA intake. Thus, in order to estimate $n$-3 PUFA intake, data on the actual intake of fish throughout Europe were collated (Table 3), and it was assumed that all fish eaten was oily fish, providing $20 \mathrm{~g} n-3$ PUFA $/ \mathrm{kg}$. Using these data, the estimated level of long chain $n$-3 PUFA con- sumption ranged from $0.07 \%$ energy to $0.64 \%$ energy. It is important to note that the assumption that all fish eaten was oily fish is a gross overestimation of actual long chain $n-3$ PUFA intake. Even so, in most European countries this estimated level of intake of long chain $n-3$ PUFA is much lower than the recommended levels.

Siskovick et al. (1995) demonstrated an inverse dosedependent relationship between fish intake and the relative risk of primary CHD. Table 4 presents the relationship between the quartiles of $n-3$ PUFA intake and the risk of CHD, and demonstrates the number of monthly portions ( $100 \mathrm{~g}$ fish per portion) of oily fish which would be required to achieve the level of $n-3$ PUFA intakes within each quartile. Thus, it is feasible to achieve $n$-3 PUFA intakes which are associated with a significantly reduced risk of CHD by increasing fish consumption. However, a large percentage (up to 65) of the population do not eat fish (Gregory et al. 1990). Clearly there is a need for alternative sources of $n-3$ PUFA, such as functional foods containing microencapsulated fish oil or genetically-modified oils whose unique fatty acids composition could fortify staple foods, thereby promoting optimal unsaturated fatty acid status.

Table 3. Current levels of long chain $n-3$ polyunsaturated fatty acid (PUFA) intake in Europe

\begin{tabular}{lcccc}
\hline Country & $\begin{array}{c}\text { Fish intake } \\
(\mathrm{g} / \mathrm{d})\end{array}$ & $\begin{array}{c}n \text {-3 PUFA } \\
(20 \mathrm{~g} / \mathrm{kg} \text { fish })\end{array}$ & $\begin{array}{c}\text { Energy intake } \\
(\mathrm{MJ} / \mathrm{d})\end{array}$ & $\begin{array}{c}n \text {-3 PUFA } \\
(\% \text { energy })\end{array}$ \\
\hline The Netherlands & 10 & 0.20 & $10 \cdot 1$ & 0.07 \\
Belgium & 17 & 0.34 & 10.4 & 0.12 \\
Germany & 16 & 0.32 & 8.8 & 0.13 \\
Ireland & 19 & 0.38 & 9.7 & 0.15 \\
Italy & 24 & 0.48 & 11.4 & 0.16 \\
Denmark & 26 & 0.52 & 11.3 & 0.17 \\
UK & 27 & 0.54 & 8.7 & 0.23 \\
Spain & 72 & 1.44 & 12.7 & 0.42 \\
Portugal & 92 & 1.84 & 10.7 & 0.64 \\
\hline
\end{tabular}

Table 4. Dietary intake of fish and the risk of primary cardiac arrest (Adapted from Siscovick et al. 1995)

\begin{tabular}{|c|c|c|c|c|c|}
\hline \multirow{2}{*}{$\begin{array}{l}\text { Variable } \\
\text { Dietary intake (g n-3 PUFA } \\
\quad \text { per month) }\end{array}$} & \multirow{2}{*}{$\begin{array}{l}\text { No } \\
\text { fish } \\
0\end{array}$} & \multicolumn{4}{|c|}{ Quartiles of fish intake } \\
\hline & & 0.96 & 2.94 & $5 \cdot 54$ & $13 \cdot 65$ \\
\hline No. of cases $(n 295)$ & 34 & 92 & 77 & 45 & 47 \\
\hline No. of controls ( $n$ 398) & 19 & 91 & 101 & 94 & 95 \\
\hline $\begin{array}{l}\text { Unadjusted odds risk } \\
\qquad(95 \% \mathrm{Cl})\end{array}$ & $1 \cdot 0$ & 0.9 & 0.7 & 0.5 & 0.3 \\
\hline $\begin{array}{l}\text { Adjusted odds risk } \\
\qquad(95 \% \mathrm{Cl})\end{array}$ & $1 \cdot 0$ & 0.9 & $0 \cdot 7$ & 0.5 & 0.4 \\
\hline \multicolumn{6}{|c|}{ Actual fish intake (portions per month) } \\
\hline \multicolumn{6}{|c|}{ Fresh trout $(9 \mathrm{~g} n-3$} \\
\hline PUFA/kg) & & 1.07 & $3 \cdot 27$ & $6 \cdot 16$ & $15 \cdot 17$ \\
\hline \multicolumn{6}{|l|}{ Tinned salmon $(13 \mathrm{~g} n-3$} \\
\hline \multicolumn{6}{|l|}{ Fresh mackerel (18g $n-3$} \\
\hline PUFA $/ \mathrm{kg}$ ) & & 0.53 & 1.63 & 3.08 & 7.58 \\
\hline
\end{tabular}

PUFA, polyunsaturated fatty acids. 


\section{References}

Ågren JJ, Hanninen O, Hanninen A \& Seppanen K (1990) Dose responses in platelet fatty acid composition, aggregation and prostanoid metabolism during moderate freshwater fish diet. Thrombosis Research 57, 565-575.

Anderson LF, Solvoll K \& Drevon CA (1996) Very-long-chain $n$-3 fatty acids as biomarkers for intake of fish and $n-3$ fatty acid concentrates. American Journal of Clinical Nutrition 64, 305-311.

Bang HO \& Dyerberg J (1972) Plasma lipids and lipoproteins in Greenlandic West-coast Eskimos. Acta Medica Scandinavica 192, 85-94.

Blonk MC, Bilo HJG, Nauta JJP, Popp-Snijders C, Mulder C \& Donker AJM (1990) Dose-response effects of fish-oil supplementation in healthy volunteers. American Journal of Clinical Nutrition 52, 120-127.

British Nutrition Foundation (1992) Recommendations for intakes of unsaturated fatty acids. Unsaturated Fatty Acids: Nutritional and Physiological Significance. The Report of the British Nutrition Foundation's Task Force, pp. 152-163. London: Chapman \& Hall.

Burr ML, Fehily AM, Gilbert JF, Rogers S, Holliday RM, Sweetnam PM, Elwood PC \& Deadman NM (1989) Effects of changes in fat, fish, and fibre intakes on death and myocardial reinfarction: diet and reinfarction trial (DART). Lancet ii, 757-761.

de Lorgeril M, Renaud S, Mamelle N, Salen P, Martin J-L, Monjaud I, Guidollet J, Touboul P \& Delaye J (1994) Mediterranean alpha-linolenic acid rich diet in secondary prevention of coronary heart disease. Lancet 343, 1454-1459.

Department of Health (1991) Dietary Reference Values for Food Energy and Nutrients for the United Kingdom. Report on Health and Social Subjects no. 41. London: H.M. Stationery Office.

Department of Health (1994) Diet and risk. Nutritional Aspects of Cardiovascular Disease. Report on Health and Social Subjects no. 46, pp. 123-144. London: H.M. Stationery Office.

Endres S, Ghorbani R, Kelley VE, Georgilis K, Lonnemann G, van der Meer JWM, Cannon JG, Rogers TS, Klempner MS, Weber PC, Schaefer EJ, Wolff SM \& Dinarello CA (1989) The effect of dietary supplementation with $n-3$ polyunsaturated fatty acids on the synthesis of interleukin-1 and tumor necrosis factor by mononuclear cells. New England Journal of Medicine 320, 265-271.

Food and Agriculture Organization/World Health Organization (1998) General conclusions and recommendations of the consultation. Expert Consultation on Fats and Oils in Human Nutrition, pp. 3-9. Rome: FAO.

Freese R \& Mutanen M (1997) $\alpha$-Linolenic acid and marine longchain $n-3$ fatty acids differ only slightly in their effects on hemostatic factors in healthy subjects. American Journal of Clinical Nutrition 66, 591-598.

Gregory J, Foster K, Tyler H \& Wiseman M (1990) The Dietary and Nutritional Survey of British Adults. London: H.M. Stationery Office.

Harris WS (1996) n-3 Fatty acids and lipoproteins: Comparison of results from human and animal studies. Lipids 31, 243-252.

Hirai A, Hamazaki T, Terano T, Nishikawa T, Tamura Y \& Kumagai A (1980) Eicosapentaenoic acid and platelet function in Japanese. Lancet i, 1132-1133.

Katan MB, van Birgelen A, Deslypere JP, Penders M \& van Staveren WA (1991) Biological markers of dietary intake, with emphasis on fatty acids. Annals of Nutrition and Metabolism 35, 249-252.

Kim DN, Eastman A, Baker JE, Mastrangelo A, Sethi S, Ross JS, Schmee J \& Thomas WA (1995) Fish oil, atherogenesis and thrombogenesis. Annals of the New York Academy of Sciences 748, 474-481.

Kromhout D, Bosschieter EB \& de Lezenne Coulander C (1985) The inverse relation between fish consumption and 20-year mortality from coronary heart disease. New England Journal of Medicine 312, 1205-1209.

Leaf A \& Weber PC (1988) Cardiovascular effects of $n-3$ fatty acids. New England Journal of Medicine 318, 549-557.

Li X \& Steiner M (1991) Dose response of dietary fish oil supplementations on platelet adhesion. Arteriosclerosis and Thrombosis 11, 39-46.

Mantzioris E, James MJ, Gibson RA \& Cleland LG (1995) Differences exist in the relationships between dietary linoleic and $\alpha$-linolenic acids and their respective long-chain metabolites. American Journal of Clinical Nutrition 61, 320-324.

Roche H \& Gibney MJ (1994) The effect of consumption of fish oil-enriched spreadable fats on platelet phospholipid fatty acid composition in human volunteers. International Journal of Vitamin and Nutrition Research 64, 237-242.

Roche HM \& Gibney MJ (1996) Postprandial triacylglycerolaemia: the effect of low-fat dietary treatment with and without fish oil supplementation. European Journal of Clinical Nutrition 50, $617-624$.

Sanders TAB, Hinds A \& Pereira C (1989) Influence of $n-3$ fatty acids on blood lipids in normal subjects. Journal of Internal Medicine 225, 99-104.

Sanders TAB, Vickers M \& Haines AP (1981) Effect on blood lipids and haemostasis of a supplement of cod-liver oil, rich in eicosapentaenoic and docosahexaenoic acids, in healthy young men. Clinical Science 61, 317-324.

Sanders TAB \& Younger KM (1981) The effect of dietary supplements of $\omega 3$ polyunsaturated fatty acids on the fatty acid composition of platelets and plasma choline phosphoglycerides. British Journal of Nutrition 45, 613-616.

Schmidt EB, Varming K, Ernst E, Madsen P \& Dyerberg J (1990) Dose-response studies on the effect of $n-3$ polyunsaturated fatty acids on lipids and haemostasis. Thrombosis Haemostasis $63,1-5$.

Scientific Committee for Food (1993) Essential fatty acids. Reports of the Scientific Committee for Food. Series no. 31, Nutrient and Energy Intakes for the European Community, pp. 52-59. Luxembourg: Commission of the European Communities.

Singh B, Niaz MA, Sharma JP, Dumar R, Rastogi V \& Moshiri M (1997) Randomized, double-blind, placebo controlled trial of fish oil and mustard oil in patients with suspected acute myocardial infarction: The Indian experiment of infarct survival - 4. Cardiovascular Drugs and Therapy 11, 485-491.

Siscovick DS, Raghunathan TE, King I, Weinamn S, Wicklund KG, Albright J, Bovbjerg V, Arbogast P, Smith H, Kushi LH, Cobb LA, Copass MK, Psaty BM, Lemaitre R, Retzlaff B, Childs M \& Knopp RH (1995) Dietary intake and cell membrane levels of long-chain $n-3$ polyunsaturated fatty acids and the risk of primary cardiac arrest. Journal of the American Medical Association 274, 1363-1367.

Wallace JM, Turley E, Gilmore WS \& Strain JJ (1995) Dietary fish oil supplementation alters leukocyte function and cytokine production in healthy women. Arteriosclerosis, Thrombosis and Vascular Biology 15, 185-189. 
https://doi.org/10.1017/S002966519900052X Published online by Cambridge University Press 